\title{
Flexible glass flat-fibre chips and femtosecond laser inscription as enabling technologies for photonic devices
}

\author{
Christos Riziotis $*^{\mathrm{a}}$, Kyriacos Kalli* ${ }^{\mathrm{b}}$, Christos Markos ${ }^{\mathrm{a}}$, Andreas Posporis ${ }^{\mathrm{b}}$, Charalambos \\ Koutsides $^{\mathrm{b}}$, Andrew S. Webb ${ }^{\mathrm{c}}$, Christopher Holmes ${ }^{\mathrm{c}}$, James C. Gates ${ }^{\mathrm{c}}$, Jayanta K. Sahu ${ }^{\mathrm{c}}$, and \\ Peter G. R. Smith \\ a Theoretical and Physical Chemistry Institute, Photonics and Nanoapplications Lab, National \\ Hellenic Research Foundation, 48 Vassileos Constantinou Avenue, 11635 Ahens, Greece; \\ ${ }^{\mathrm{b}}$ Nanophotonics Research Laboratory, Cyprus University of Technology, Lemessos, Cyprus; \\ ${ }^{\mathrm{c}}$ Optoelectronics Research Centre, University of Southampton, Southampton SO17 1BJ, UK
}

\begin{abstract}
The potential of a new class of multifunctional photonic circuits is demonstrated by femtosecond laser micro-machining and inscription of micro-optical structures, such as ring and disk resonators, Mach-Zehnder interferometers, and microfluidic devices, in a flexible flat-fibre chip. Additional filling of surface channels with functional materials is employed towards sensing applications. Furthermore, direct write femtosecond-laser inscribed Bragg gratings were written in the Ge-doped core of flat-fibre enabling a number of relevant applications. The flat-fibre platform offers a unique degree of freedom by allowing surface and sub-surface devices to be integrated onto an a single optical chip with the potential for straightforward incorporation into integrated photonic circuits or optofluidic devices.
\end{abstract}

Keywords: Femtosecond laser inscription, optical fibres, flat fibre, integrated optics, microfluidics, sensors

\section{INTRODUCTION}

There is immense interest in developing enabling technologies for potential use in smart structures, integrated photonic circuits and devices for use in optofluidics, and where the availability of a flexible, optically-flat, glass fibre would offer a means to incorporate all the advantages of densely packed planar chips, with the many advantages afforded by optical fibres. A waveguiding glass substrate or flat optical fibre chip is inherently compatible with optical fibres, sharing all the advantages of the cylindrical glass optical fibres, such as the ability to withstand high ambient temperatures, a chemically inert host material, but having a large flat surface area allowing for material processing. Coupled with low manufacturing costs, these flexible chips can prove to be a key technology applied to disposable high-end sensing devices or fully distributed point sensors.

Here we present experimental investigations into creating novel structures (such as microfluidic channels, ring resonators, etc.) using a direct write approach and femtosecond laser micromachining on this new flat-fibre platform. We have successfully demonstrated the use of femtosecond laser micro-machining and inscription of different microoptical structures, such as ring and disk resonators, Mach-Zender interferometers, in addition to a complex microfluidic devices, in a flat fibre optical chip. The inscribed structures are subsequently filled with active polymers and other materials that could be employed for various applications. Furthermore, direct write femtosecond-laser inscribed Bragg gratings have been written in the Ge-doped core of the flat-fibre. The morphology of the fabricated structures has been characterized using optical microscopy and 3D optical profilometry.

*riziotis@eie.gr, phone +302107273887,www.eie.gr.

*kyriacos.kalli@cut.ac.cy; phone 35725002609; www.cut.ac.cy 


\section{THE FLAT FIBRE PHOTONIC PLATFORM}

Flat fibre ${ }^{1}$ samples were provided by the Optoelectronics Research Centre, University of Southampton and they exhibited a planar germanosilicate core of $\sim 8 \mu \mathrm{m}$ height, in order to have a good mode matching with the conventional SMF-28, and also single mode operation at $1550 \mathrm{~nm}$. The width of the samples measured to be $\sim 1.1 \mathrm{~mm}$. The cross section of the initial unpolished fiber is shown in Figure 1 and had thickness of $390 \mu \mathrm{m}$.
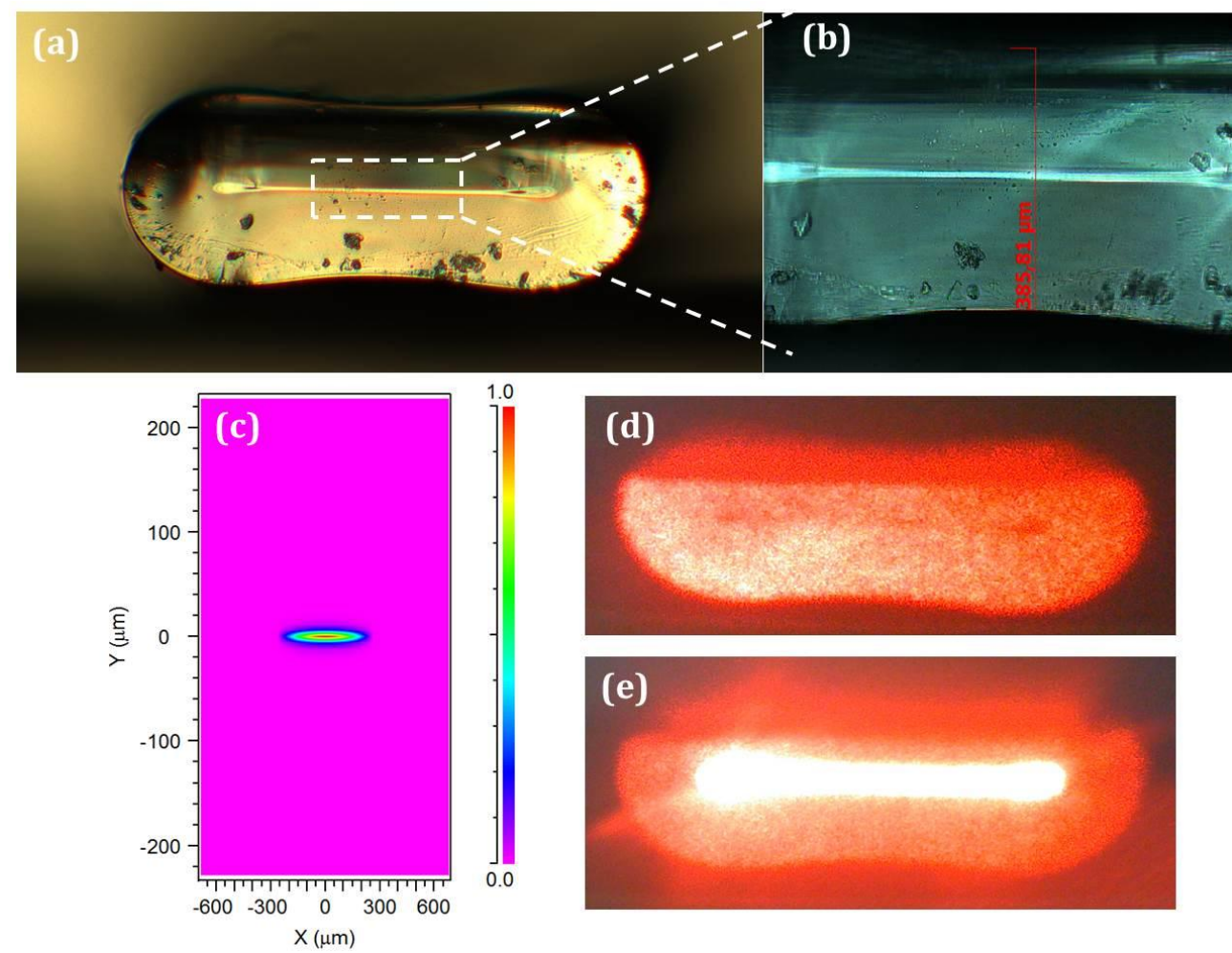

Figure 1. (a) Optical image of the cross-section of flat fiber using 4x objective. (b) Optical image focused on the core of the fiber using 20x microscope objective. (c) Calculated fundamental guiding mode at $1.55 \mu \mathrm{m}$ wavelength. (d) Near-field image of the fiber out of light coupling and (e) in light coupling

Using a commercially available mode solver (RSoft BPM) the fundamental guiding mode of the flat fiber was calculated to exhibit an effective refractive index $\mathrm{n}_{\mathrm{eff}}=1.45068$. The mode profile is shown in Figure 1(c) while Fig. 1 (d) and (e) shows the end facet of the fiber before and after coupling the light to the core of the fiber. It should be emphasized that the fiber exhibited single mode guidance at $1550 \mathrm{~nm}$. However, the capture of the near-field pattern of the fundamental mode was not possible without an IR camera.

In order to have easier access to the core the fiber, we also used modified flat fibers polished down to $\sim 45 \mu \mathrm{m}$ distance from the planar core. In Figure 2 below it is shown the cross-section of the polished fiber we also used in our experiments. Samples were glued in a microscope slide to avoid any undesired movement, and mounted in a single XYZ stage during the inscription process. The lengths of all the used samples (both polished and unpolished) were about $\sim 1-2$ cm long. 

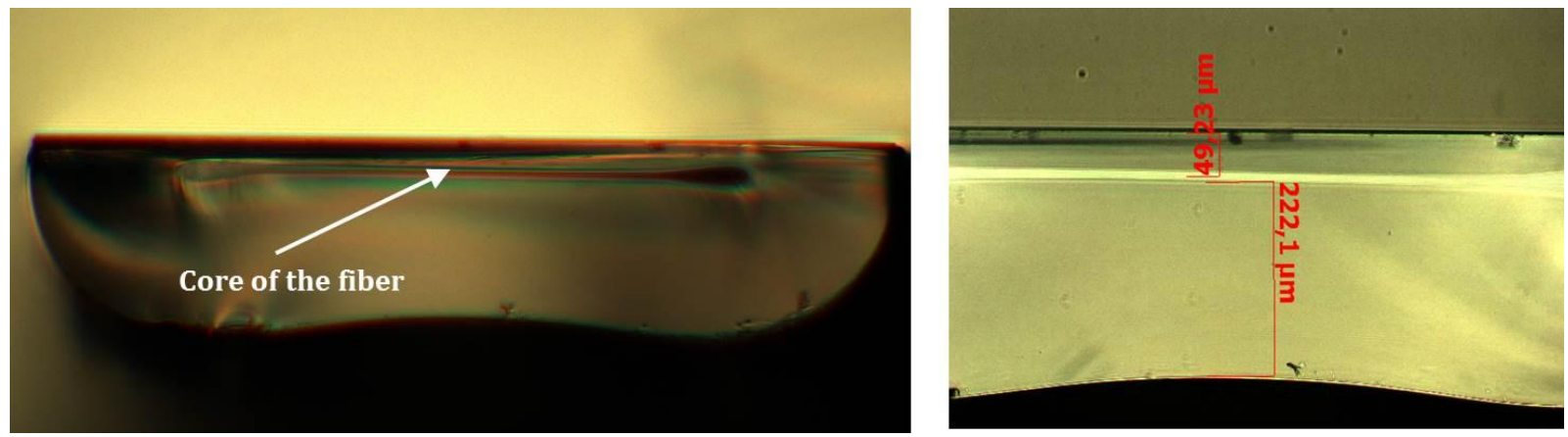

Figure 2. Cross section of the polished flat-fiber with remaining overcladding of approximately $50 \mu \mathrm{m}$ from the center of core.

\section{EXPERIMENTAL}

This section presents the experimental results from the femtosecond lased based inscription of several structures in flatfibre demonstrating the potential for the integration of many diverse optical devices such as ring and disk resonators, Mach Zehnder interferometers, and Bragg gratings in combination eventually with microfluidic channels. Structures were written with different condition and at varying depths. Surface structures and channels were also filled with different materials like PDMS (Polydimethylsiloxane) and PVK (poly-N-vinyl carbazole) polymers with a modified dipcoating technique.

\subsection{Femtosecond laser micromachining system}

The femtosecond laser inscription system ${ }^{2}$ is based on an infrared femtosecond laser with $~ 300 \mathrm{fs}$ laser pulses at $\sim 1030 \mathrm{~nm}$ wavelength. The repetition rate of the laser can be adjusted from $1-100 \mathrm{kHz}$ according to user requirements. The laser beam passes through a shutter and is focused with a 50x objective lens. Using a CCD camera, we could monitor in real-time the inscription of the structure at the surface of the silica substrate or flat-fiber. The sample (flatfiber) was mounted on a silica substrate (perpendicular to the machining lens) and then placed in an XYZ micro-position stage and subsequently on a subnanometer precision XY air-bearing stage system (Aerotech). The $\mathrm{Z}$ axis of the system could be controlled using another highly precise translation stage

\subsection{Ring resonator inscription in the flat-fiber}

A number of ring resonators, sub-surface and also on the surface of flat fibre were inscripted in polished samples. The following structures are created at the edge of the fiber where there is the possibility of filling the structures with an active material afterwards. Figure 3 shows two resonators structures fabricated by different number of writing steps, the first (top) resonator by one step writing while the other by 10 repeats of inscription. The widths of the channels are $\sim 10 \mu \mathrm{m}$ and the diameter of the ring $100 \mu \mathrm{m}$. The number of repeats affects the inscription width and despite the fact that the pre-default distance between the channel and the ring was defined at $5 \mu \mathrm{m}$, it is clear that for the case of 10 repeats the channel collapsed with the ring creating different features. The energy used for the inscription was $\sim 6.7 \mu \mathrm{J} / \mathrm{pulse}$ at $100 \mathrm{kHz}$ repetition rate while the inscription speed was $50 \mu \mathrm{m} / \mathrm{sec}$. The measured depth of the first resonator was $\sim 9 \mu \mathrm{m}$ while in the second resonator was $\sim 15 \mu \mathrm{m}$. 


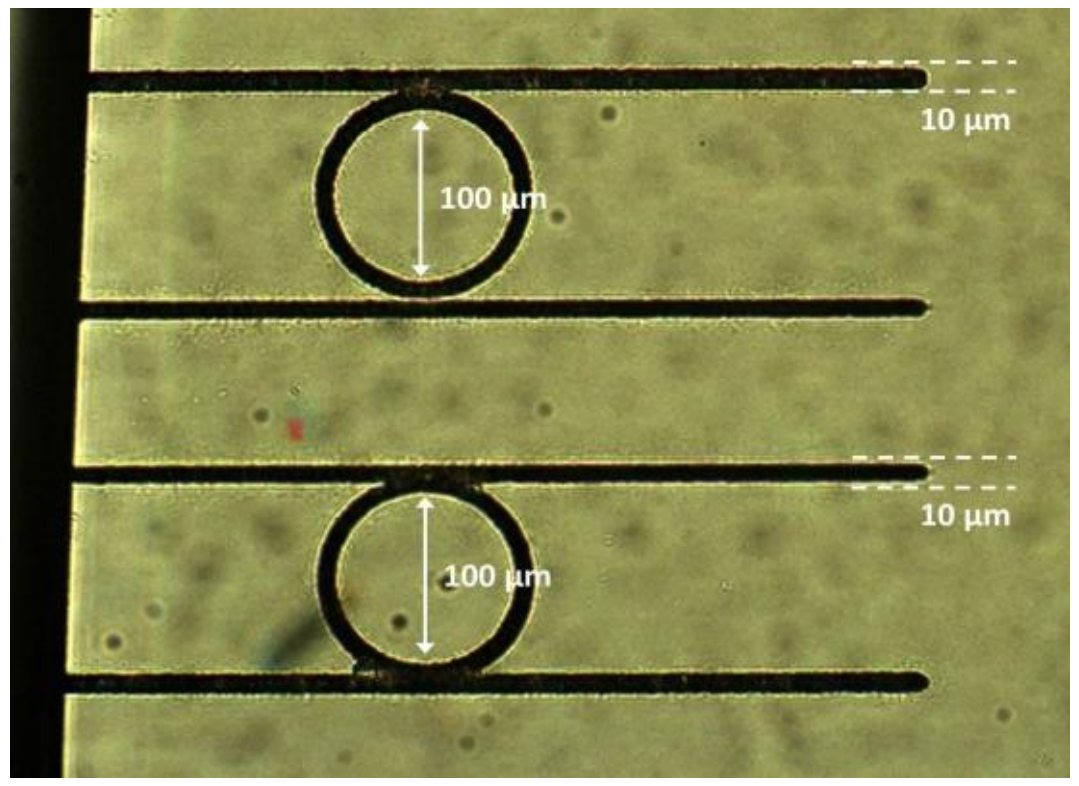

Figure 3. Two ring resonators in the top surface of the fiber inscripted with different number of writing steps

For the second ring structure with the collapsed channel-ring separation section it was performed 3D profilometer measurements, after filing the structure with water in order to assess structure's geometrical properties.

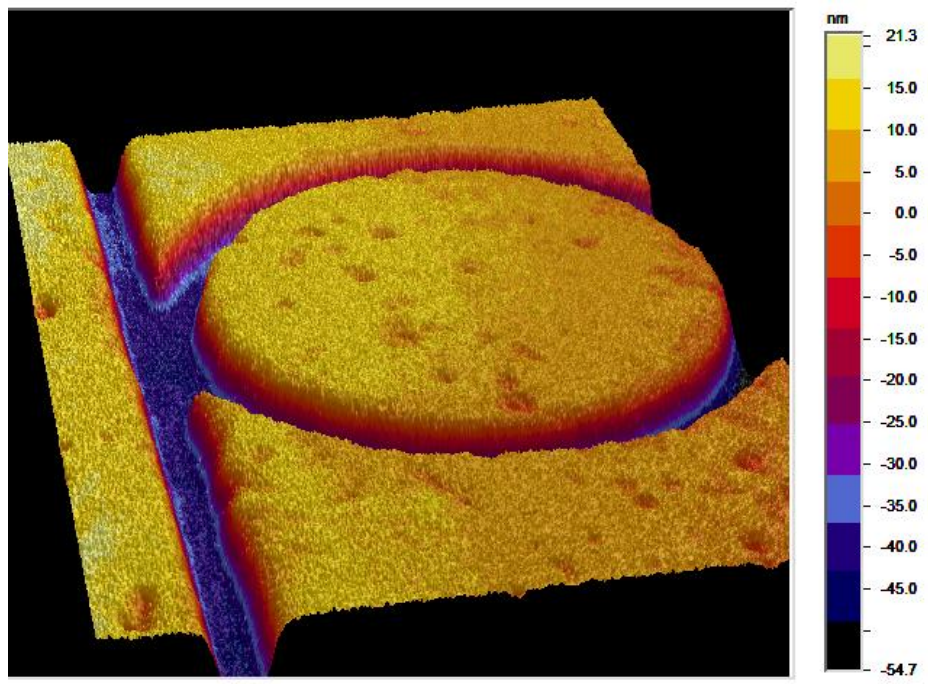

Figure 4. View of inscribed ring resonator when partially filled with water.

Furthermore, we inscribed five cascaded ring resonators starting the inscription from the surface of the fiber going down to the cladding of the fiber at depths of 5, 10 and $20 \mu \mathrm{m}$ in order to investigate the possibility of creating the structures several microns under the surface. The following Figure 5 shows optical images (all focused on the surface of the fiber) of the inscribed resonators at different depths. The last two resonators (bottom image) show the difference between the 5 and 10 times repeat of inscription. 

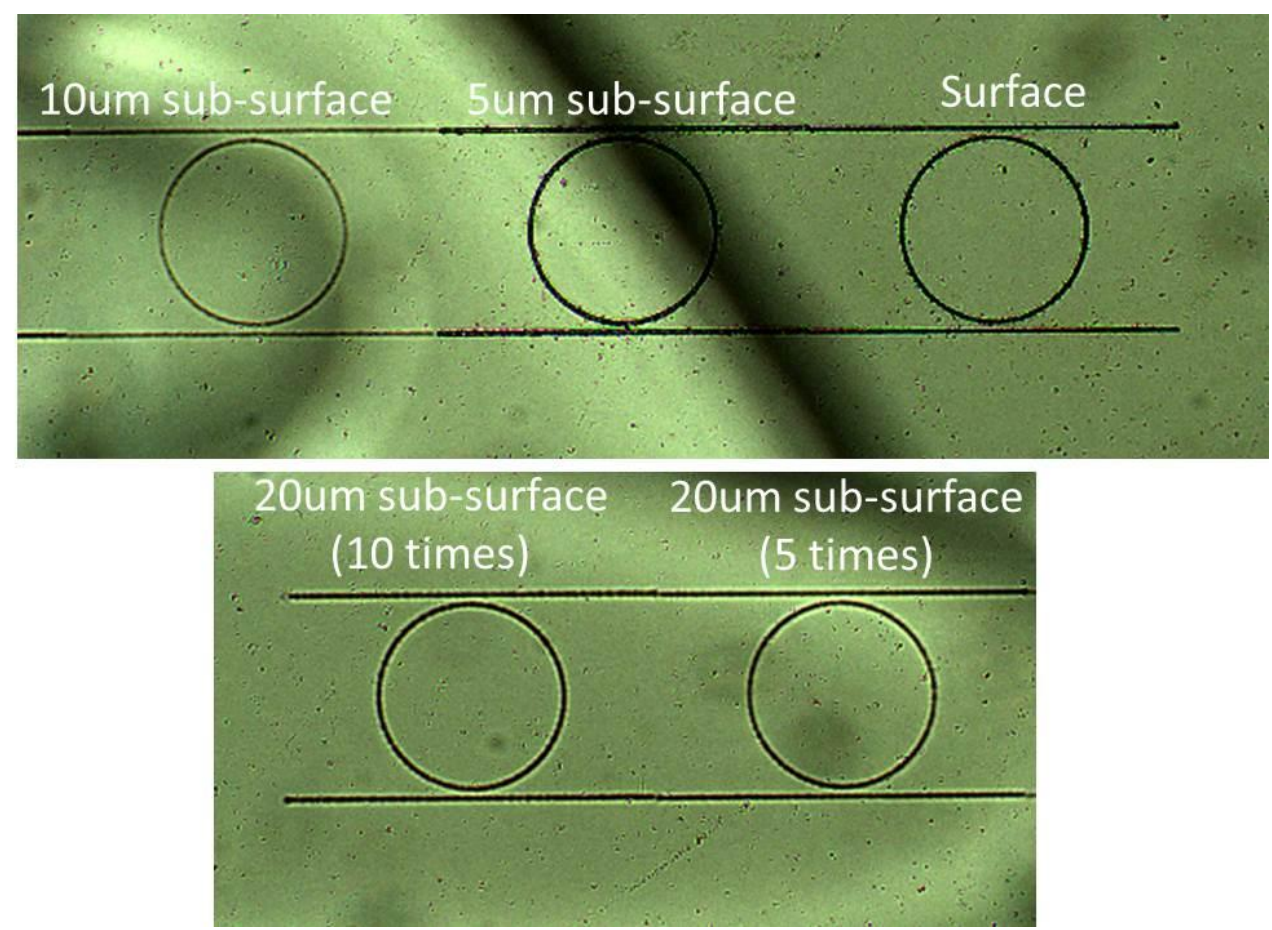

Figure 5. Inscription of 5 cascaded ring resonators at different inscription depths (surface, 5, 10 and $20 \mu \mathrm{m}$-sub-surface). The last two resonators inscribed 5 times and 10 times respectively.

\subsection{Disk resonator inscription}

By creating many homocentric circles with decreasing diameter and in a very short distance between them in a such way of overlapping, it was also fabricated a disk resonator structure. All the parameters of the laser and the characteristics of the disk remained the same as for the ring resonators structures writing.. Figure 6 shows the optical image of the disk resonator and the corresponding 3D image from the profilometer. Filling the disk area with sensitive materials of appropriately high refractive index could lead to resonant type sensing devices on the flat fibre.
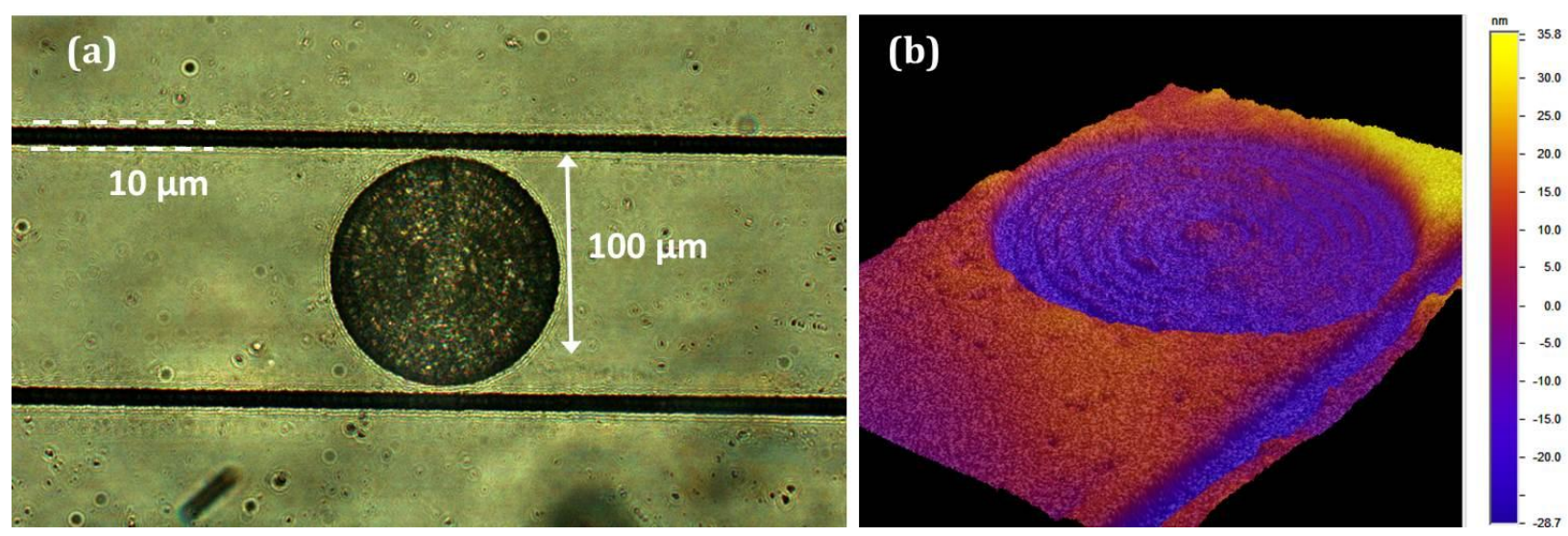

Figure 6. (a) Optical image of the disk resonator. (b) 3D image from the profilometer. 


\subsection{Mach-Zehnder interferometer inscription}

Another structure of increased importance for sensing or telecommunication applications is the Mach-Zehnder interferometer. Below in Figure 7 it is shown the optical microscope images of two Mach-Zehnder interferometers defined on the top surface of the fibre. The laser energy was $3.5 \mu \mathrm{J} / \mathrm{pulse}$ and the speed of inscription $50 \mu \mathrm{m} / \mathrm{sec}$. The 3D image from the profilometer indicates smooth transition to the two arms of the Mach-Zender. Furthermore, by repeating the inscription several times it was achieved a better quality channel, free from any undesired debris material. It should be noted that those channels were filled with PDMS, in order to verify that the drop-casting technique is a proper method for filling the micro machined channels with the desired material each time, suitable for specific applications ${ }^{3}$.
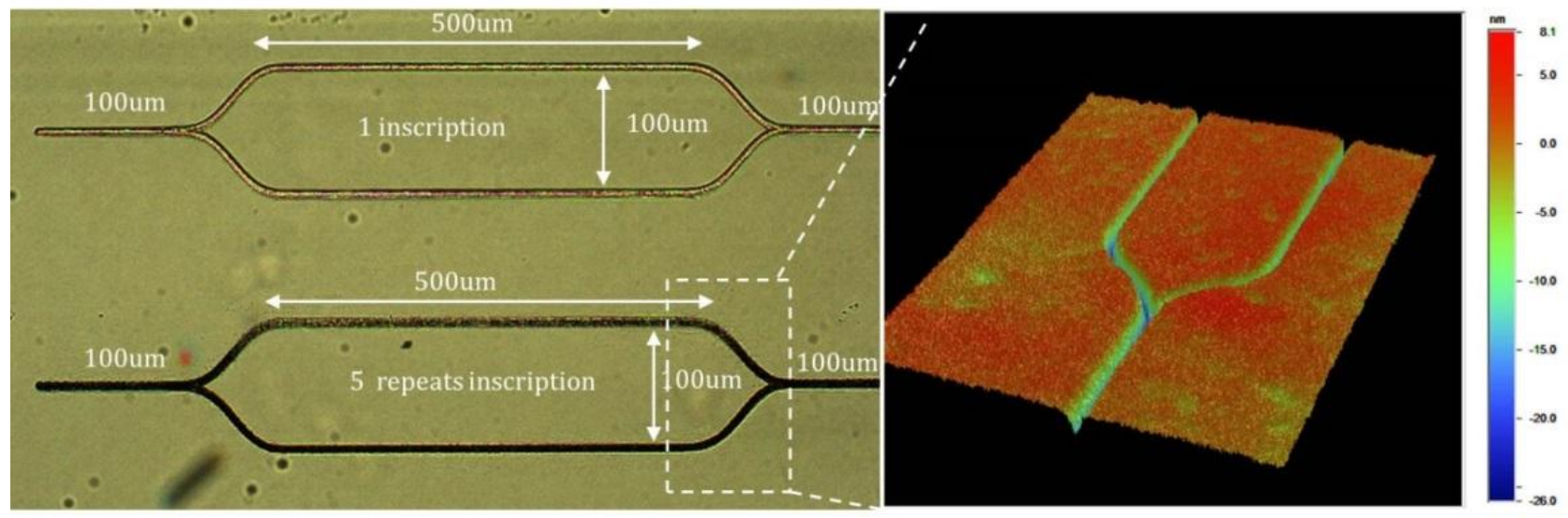

Figure 7. Optical images of two Mach-Zender interferometers. The top structure made using 1 inscription while the bottom with 5 repeats. Profilometer 3D image demonstrating the structures' quality (Right)

\subsection{Direct Bragg grating inscription in flat-fibre's planar core}

Here is presented also the inscription of a $2^{\text {nd }}$ order Bragg Grating in a surface polished flat-fibre using femtosecond laser pulses. The pulse energy was reduced down to $1.6 \mu \mathrm{J} /$ pulse and the repetition rate was changed to $1 \mathrm{kHz}$. Figure 8 shows the response of a Bragg grating inscribed in the Ge-doped core of the fiber that was $33 \mu \mathrm{m}$ under the polished side of the fibre. The length of the grating was around $8 \mathrm{~mm}$ and the period $\sim 1.1 \mu \mathrm{m}$.

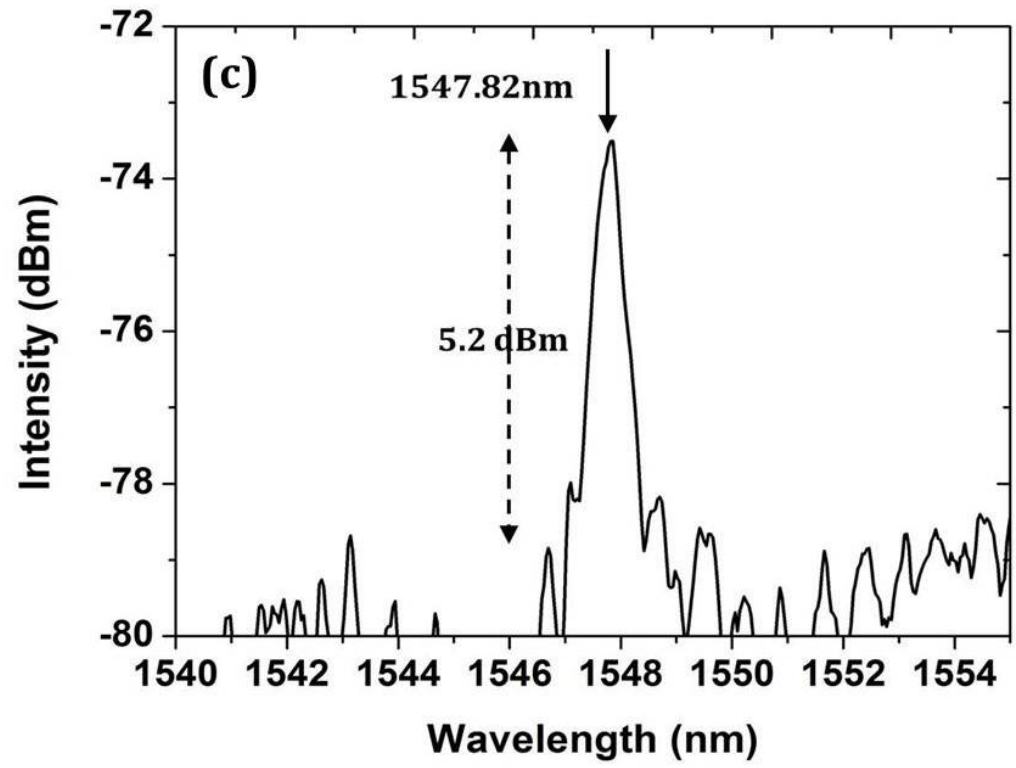

Figure 8. Bragg grating spectrum of the flat fibre. 


\subsection{Fabrication of microfluidic structures}

Several surface microfluidic channels and structures were also fabricated as a demonstration of the potential of the flat fiber to accommodate such diverse and complex structures. The design of the microfluidic device constitutes of three inputs, a sinewave-like channel for the delay of the fluids and a block of channels for liquid mixing. The laser energy was $6.7 \mu \mathrm{J} / \mathrm{pulse}$ at $100 \mathrm{kHz}$ repetition rate.

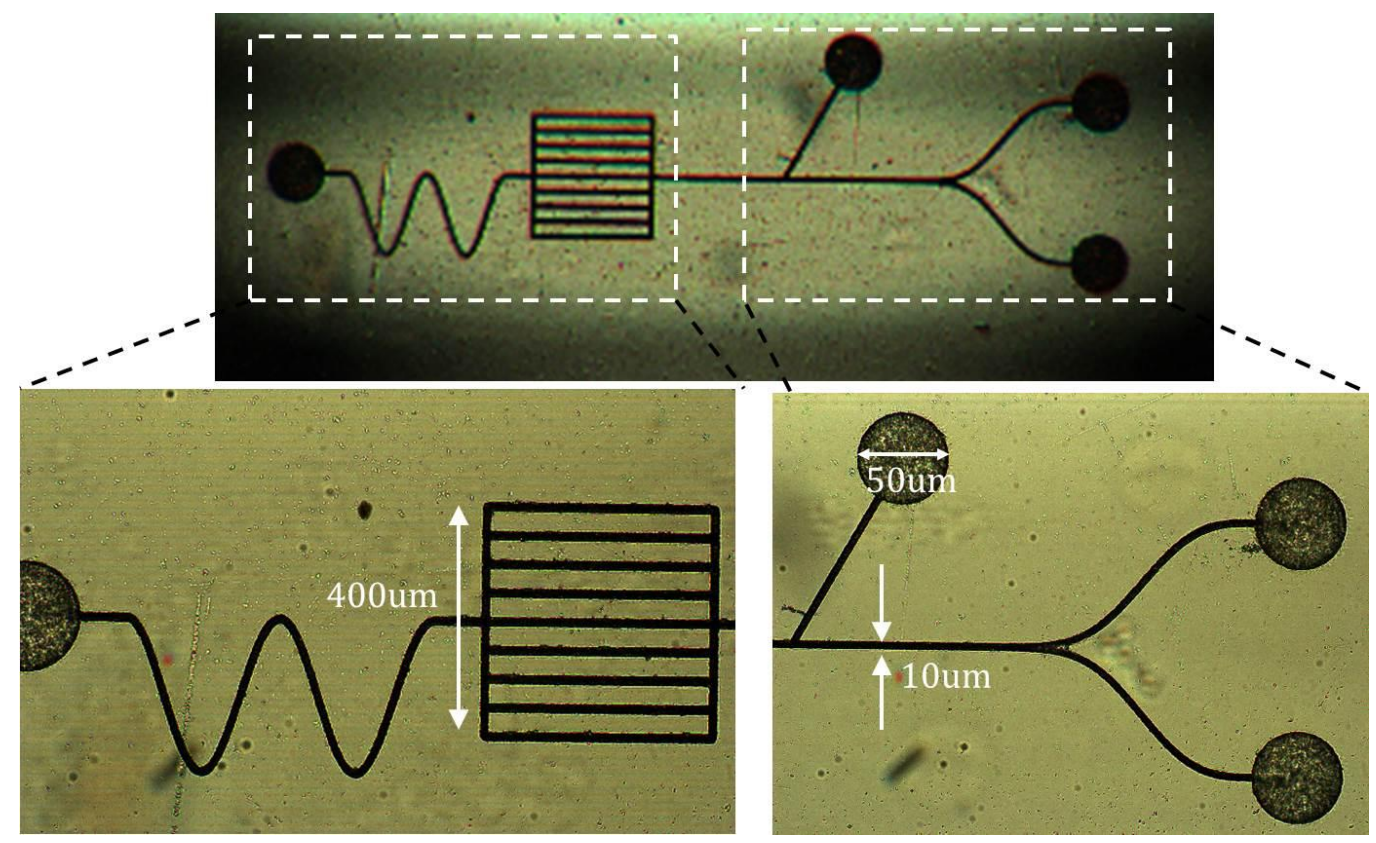

Figure 9. Optical images of the microfluidic device on the top surface of the flat- fiber.

\section{CONCLUSIONS}

Flat fibre appears to be a promising new optical platform combining the advantages of flexible long length silica fibers together with increased lateral and vertical dimensions allowing thus the development of combined integrated circuits with enhanced functionality. By the use of femtosecond laser micromachining and direct writing techniques we presented experimental investigations towards the development of integrated structures such as microfluidic channels, ring resonators and disks, Mach-Zehnder interferometers' and also Bragg gratings in flat fibres. The availability of such diverse photonic structures in a single optical chip could allow the development of advanced devices, and especially microfluidic based biosensing systems as they could be supported by the presence of those ultra sensitive resonant structures acting as detection/response interrogators, over a relatively long length available by the flat fibre.

\section{ACKNOWLEDGENMENTS}

National Hellenic Research Foundation acknowledges financial support from R\&D Project WelCOM 09SYN-71-856 funded by National Strategic Reference Framework NSRF 2007-2013/Hellenic General Secretariat for Research and Technology - GSRT. The authors acknowledge funding from the COST action TD1001 (STSM reference number: COST-STSM-ECOSTSTSM-TD1001-270513-031731). Cyprus University of Technology acknowledges the Research

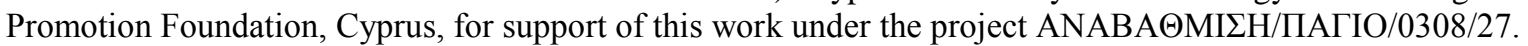




\section{REFERENCES}

[1] Adikan, F. R. M., Sandoghchi S., Chong, W., Simpson, R., Mahdi, M., Webb, A., Gates, J., Holmes, C., "Direct UV written optical waveguides in flexible glass flat fibre chips," IEEE J. Sel. Topics Quantum Electron., 18(5), 1534-1539 (2012).

[2] Smith, G. N., Kalli, K., and Sugden, K., "Advances in femtosecond machining and inscription of micro and nano photonic devices," [Frontiers in Guided Wave Optics and Optoelectronics] (2010)

[3] Athanasekos, L., El Sachat, A., Pispas, S., and Riziotis, C., "Amphiphilic diblock copolymer based multiagent photonic sensing scheme," Journal of Polymer Science Part B: Polymer Physics 52(1), 46-54 (2014). 